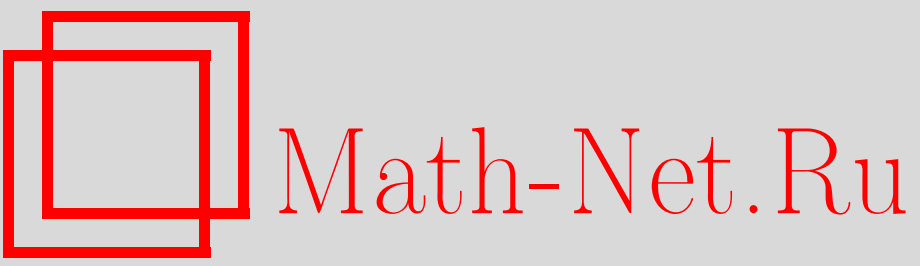

O. V. Lukashenko, E. V. Morozov, M. Рagano, Об эффективности оценки Монте Карло на основе гауссовского моста, Информ. и её примен., 2017, том 11, выпуск 2, 16-24

DOI: https://doi.org/10.14357/19922264170202

Использование Общероссийского математического портала Math-Net.Ru подразумевает, что вы прочитали и согласны с пользовательским соглашением

http://www. mathnet.ru/rus/agreement

Параметры загрузки:

IP: 54.198 .55 .26

26 апреля 2023 г., 10:22:39 


\title{
ON THE EFFICIENCY OF BRIDGE MONTE-CARLO ESTIMATOR
}

\author{
O. V. Lukashenko ${ }^{1}$, E. V. Morozov ${ }^{2}$, and M. Pagano ${ }^{3}$

\begin{abstract}
Long-term correlation is a key feature of traffic flows and has a deep impact on network performance. Indeed, the arrival rate can persist on relatively high values for a considerable amount of time, provoking long busy periods and possibly bursts of lost packets. The authors focus on Gaussian processes, well-recognized and flexible traffic models, and consider the probability that the normalized cumulative workload grows at least as the length $T$ of the considered interval. As $T$ increases, such event becomes rare and ad-hoc techniques should be used to estimate its probability. To this aim, the authors present a variant of the well-known conditional Monte-Carlo (MC) method, in which the target probability is expressed as a function of the corresponding bridge process. In more detail, they derive the analytical expression of the estimator, verify its effectiveness through simulations (for different sets of parameters), and investigate the effects of the discretization step.
\end{abstract}

Keywords: Gaussian processes; conditional Monte Carlo; bridge process; rare events; variance reduction

DOI: $10.14357 / 19922264170202$

\section{Introduction}

Key features of traffic patterns in modern computer networks are the high level of statistical multiplexing and, at the same time, strong correlations over several timescales [1]. In this framework Gaussian processes have emerged as well-recognized and flexible models able to describe the traffic dynamics of a wide class of networks $[2,3]$. Indeed, they permit to capture, in a simple and parsimonious way, the properties of self-similarity and long-range dependence, which have a deep impact on network dimensioning and QoS (Quality of Service) issues [4]. In a nutshell, self-similarity means that the distribution of the process remains unchanged under suitable scaling of time and space, while long-range dependence (also known as Joseph effect) [5] implies a slow decay of the autocorrelation function.

Network performance are, in general, deeply influenced by packet losses and many works have been devoted to the estimation of the overflow probability in presence of long-range dependent traffic (see, for instance, [3] and references therein). However, not only the loss rate is relevant, but also the way in which losses are distributed over time. Indeed, bursts of losses can significantly degrade the QoE (Quality of Experience) in case of real-time multimedia applications and also affect the throughput of elastic applications, since TCP congestion control [6] poorly reacts in presence of multiple losses during the same congestion window, which often lead to the expire of timeouts (instead of the AIMD (Additive Increase Multiplicative Decrease) behavior that happens when losses are detected via triple duplicate acknowledgements). Such bursts of losses are often determined by high-activity periods that last for relative long intervals of time, a typical consequence of the above-mentioned Joseph effect.

Moreover, the properties of long-memory and selfsimilarity make difficult the theoretical analysis even for simple queuing systems and, as a consequence, simulation is often the only available tool to investigate network performance.

Simulation permits to study the performance of complex systems with an arbitrary level of detail, but the traditional approach, known in the literature as crude $\mathrm{MC}$, becomes highly inefficient when the event of interest gets rarer and rarer. Indeed, given a required level of accuracy (typically expressed in terms of relative error), the length of the simulation is inversely proportional to the target probability, which can assume (for instance, in the case of high-quality video flows) values of the order of $10^{-9}$ [7]. Moreover, every estimate may be related to the simulation of complex networks and so includes the generation of a huge amount (of the order of millions or more, depending on the time horizon and the complexity of the system) of random numbers, with additional concerns related not only to the length of the simulation, but also to the goodness of the random generator itself.

Variance reduction techniques aim at achieving the desired accuracy with a lower number of samples [8],

\footnotetext{
${ }^{1}$ Institute of Applied Mathematical Research of Karelian Research Centre of the Russian Academy of Sciences, 11 Pushkinskaya Str., Petrozavodsk 185910, Republic of Karelia, Russian Federation; Petrozavodsk State University, 33 Lenin Str., Petrozavodsk 185910, Republic of Karelia, Russian Federation, lukashenko@krc.karelia.ru

${ }^{2}$ Institute of Applied Mathematical Research of Karelian Research Centre of the Russian Academy of Sciences, 11 Pushkinskaya Str. Petrozavodsk 185910, Republic of Karelia, Russian Federation; Petrozavodsk State University, 33 Lenin Str., Petrozavodsk 185910, Republic of Karelia, Russian Federation; emorozov@karelia.ru

${ }^{3}$ University of Pisa, 43 Lungarno Pacinotti, Pisa 56126, Italy; m.pagano@iet.unipi.it
} 
but require some additional information about the behavior of the system, typically provided (although in an asymptotic and eventually approximate form) by the Large Deviation Theory (LDT) [9]. Among them, Importance Sampling (IS) is probably the most popular approach [10] due to its links with LDT ${ }^{1}$, providing a solid theoretical background for its applicability. However, under an improper choice of the change of measure (the optimal choice is known just for simple queuing systems), the variance may even grow infinitely [11].

In this paper, based on its predecessors [12,13], the focus is on an alternative approach, known as Conditional MC, in which the target probability is expressed as a conditional expectation. Although conditional MC always leads to variance reduction, it is often impossible, or at least very difficult, to find a suitable conditioning quantity. However, in case of Gaussian processes, the target probability can be easily expressed as a function of the corresponding bridge process, as originally proposed in [14] under the name of Bridge Monte Carlo (BMC), for the estimation of the overflow probability. As mentioned above, in this work, the authors investigate the applicability of BMC to the tail distribution of the duration of high activity periods, which indeed become rare events when the duration of the considered interval goes to infinity. In comparison with the preliminary work [12], the experimental results have been widely extended: indeed, the authors investigated the asymptotic properties of the estimator, compared its performance with a basic version of IS, and analyzed the effect of the discretization step on the estimated probability.

The rest of the paper is organized as follows. In Section 2, the authors formally define the problem addressed in this work and recall the few available asymptotic results. Then, Section 3 addresses the general issues related to rare event simulation, including the basic definitions about simulation efficiency and a short description of (single-twist) IS, the most widely used variance reduction technique that will be considered later for performance comparison. The use of the bridge process is investigated in Section 4, while its performance is analyzed in Section 5, taking into account the effect of different parameters (such as the length of the activity period, the conditioning point, and the discretization step). Finally, Section 6 ends the paper with some final remarks.

\section{High Activity Periods for Gaussian Processes}

In traffic modeling, Gaussian processes have emerged as a flexible and powerful tool, able to take into account the long memory properties of real traffic, while keeping a relatively simple and elegant description.

In this work, a centered Gaussian process with stationary increments $\left\{X_{t}, t \in \mathbb{R}_{+}\right\}$is considered. Let us denote by $v_{t}:=\operatorname{Var} X_{t}$ the variance of $X_{t}$; then, the covariance function has the following expression:

$$
\Gamma_{s, t}=\frac{1}{2}\left(v_{t}+v_{s}-v_{|t-s|}\right) .
$$

It is interesting to estimate the following probability:

$$
\pi(T):=\mathbb{P}\left(\forall t \in \mathbb{T}: X_{t}>t\right)
$$

where $\mathbb{T}=(0, T] \subseteq \mathbb{R}_{+}$. Such probability is closely related to the duration of busy periods and plays an important role in the study of QoS indexes since it takes into account bursts of losses (for more details, see $[15,16]$ ).

It is worth mentioning that the present approach only requires that $v_{t}$ is an increasing function. Such condition is quite general and holds, for instance, for the following processes, well-known in the literature and widely-used in traffic modeling:

(1) fractional Brownian Motion (FBM), one of the most studied self-similar long-range dependent Gaussian processes, originally proposed in the traffic modeling framework by Norros [2]. It has been shown in [17] that FBM arises as the scaled limit process when the cumulative workload is a superposition of on-off sources with mutually independent heavy-tailed on and/or off periods. In this case,

$$
v_{t}=t^{2 H}, \quad H \in(0,1),
$$

and in the teletraffic framework, usually, $H$ $\in(1 / 2,1)$, corresponding to processes with longrange dependence;

(2) sum of independent FBMs:

$$
v_{t}=\sum_{i} t^{2 H_{i}}
$$

The use of this model is also motivated by the fundamental result in [17] in case of heterogeneous on-off sources; and

(3) integrated Ornstein-Uhlenbeck process (IOU):

$$
v_{t}=t+e^{-t}-1
$$

is the Gaussian counterpart of the well-known Anick-Mitra-Sondi fluid model [18], and its relevance in the framework of teletraffic is also discussed in [19].

\footnotetext{
${ }^{1}$ On one side, several proofs in LDT are based on IS arguments and, on the other side, efficient changes of measure are often related to sample-path LDT results
} 
Note that the analytical expression of the target probability is not known in explicit form for a general Gaussian input (including the considered examples). Indeed, there are only a few asymptotic results available, based on LDT. For instance, in the case of FBM input,

$$
\begin{aligned}
\lim _{T \rightarrow \infty} \frac{1}{T^{2-2 H}} \log \mathbb{P}\left(\forall t \in(0, T]: X_{t}>t\right) & \\
=\lim _{n \rightarrow \infty} \frac{1}{n} \log \mathbb{P}(\forall t & \left.\in(0,1]: \frac{X_{t}}{\sqrt{n}}>t\right) \\
& =-\inf _{f \in \mathscr{B}} \Lambda(f):=-\nu
\end{aligned}
$$

where

$$
\mathscr{B}:=\{f \in \mathcal{R}: f(r)>r, \forall r \in(0,1]\} ;
$$

$\Lambda$ denotes the rate function; and $\mathcal{R}$ is the reproducing kernel Hilbert space (RKHS) associated with the distribution of FBM (for more details, see [20]). Moreover, it is known [15] that the constant $\nu \in\left[1 / 2, c_{H}^{2} / 2\right]$, where

$$
\begin{aligned}
c_{H}:=[H(2 H-1) & (2-2 H) \\
& \left.\times \mathrm{B}\left(H-\frac{1}{2}, 2-2 H\right)\right]^{-1 / 2}
\end{aligned}
$$

and $B$ is the Beta function. Note that the upper bound for this constant is close to $1 / 2$ in case when $H>1 / 2$ (Fig. 1). A further characterization of the most likely path in the set $\mathscr{B}$ has been found in [21], and since an explicit expression for $\nu$ is not available, numerical methods to calculate $\nu$ have been proposed. The above-mentioned asymptotic result has been generalized in [22] to the case of Gaussian processes with regularly varying at infinity variance, which includes the sum of independent FBMs and IOU as well.

Due to the lack of exact analytical results, simulation is the only available tool for estimating the target probability (1). On the other hand, when $T \rightarrow \infty$, the event

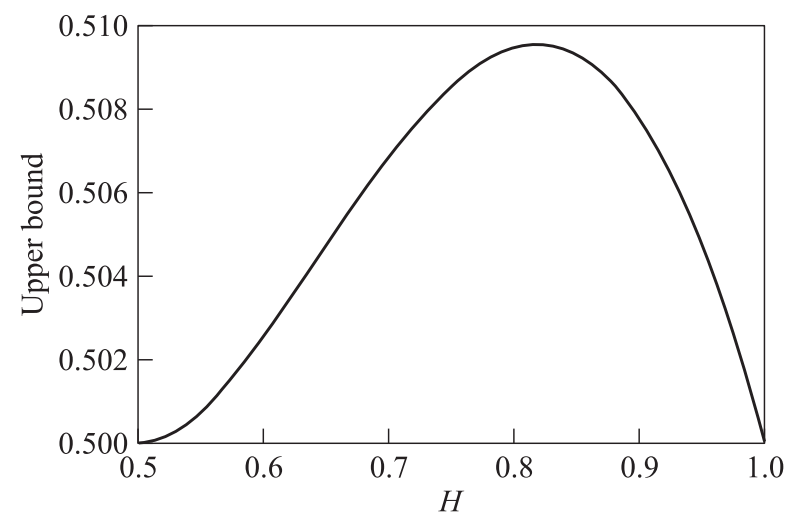

Figure 1 Upper bound for $\nu$ $\left\{\forall t \in \mathbb{T}: X_{t}>t\right\}$ becomes rare and, hence, standard $M C$ requires an unacceptable large number of generated sample paths. Indeed, the key contribution of this work is the application of a variant of the conditional MC method for variance reduction.

\section{Preliminaries on Rare Event Simulation}

Let $X$ be a random process. Consider estimating the probability

$$
\pi(T):=\mathbb{P}\left(X \in A_{T}\right)=\mathbb{E} I\left(X \in A_{T}\right)
$$

for some Borel set $A_{T}$ of the paths of the process $X$ where $I$ denotes the indicator function and $T$ is the so-called parameter of rarity: $\pi_{T} \rightarrow 0$ as $T \rightarrow \infty$. To estimate $\pi_{T}$ by standard MC simulation, one should generate $N$ replications $X^{(1)}, \ldots, X^{(N)}$ of the process $X$. In the following, there will be considered the estimators of the form

$$
\tilde{\pi}_{N}(T)=\frac{1}{N} \sum_{n=1}^{N} F_{T}\left(X^{(n)}\right)
$$

for a measurable function $F_{T}$. If $F_{T}(X)=I\left(X \in A_{T}\right)$, one has the crude MC estimator. The relative error (RE) of the estimate $\tilde{\pi}_{N}(T)$ is defined as

$$
\operatorname{RE}(\tilde{\pi}(T)):=\frac{\sqrt{\operatorname{Var}\left[\widetilde{\pi}_{N}(T)\right]}}{\mathbb{E}\left[\widetilde{\pi}_{N}(T)\right]},
$$

and for the crude estimate, it behaves as

$$
\operatorname{RE}(\widetilde{\pi}(T)) \sim \frac{1}{\sqrt{\pi(T) N}} \text { as } \pi(T) \rightarrow 0 .
$$

Therefore, the RE of the standard MC estimation is unbounded when the event becomes rare. That is why for the rare event simulation, it is crucially important to define modified estimators in order to reduce variance (and, as a result, RE).

Let us consider the number of sample paths required to obtain some given maximal RE:

$$
N_{T}=\inf \left\{N \in \mathbb{N}: \operatorname{RE}(\widetilde{\pi}(T)) \leq \mathrm{RE}_{\max }\right\}
$$

and let us introduce the concept of relative efficiency

$$
R_{T}:=\frac{\log \mathbb{E}\left[F_{T}(X)^{2}\right]}{\log \mathbb{E}\left[F_{T}(X)\right]} .
$$

An estimate (3) is said to be asymptotically optimal $[23,24]$ with respect to the parameter $T$ if

$$
\limsup _{T \rightarrow \infty} \frac{1}{T} \log N_{T}=0,
$$


i. e., if the corresponding RE increases slower than any exponential function. The latter condition is equivalent to

$$
\lim _{T \rightarrow \infty} R_{T}=2 .
$$

Remark that the limit above is always less or equal 2 since $\mathbb{E}\left[F_{T}(X)^{2}\right] \geq\left(\mathbb{E}\left[F_{T}(X)\right]\right)^{2}$. The proof of asymptotic optimality usually relies on LDT, namely, on Varadhan's lemma [23].

Let us briefly describe the method of simulation by conditioning, known in the literature as conditional $M C$ [8]. Denote

$$
Z=I\left(X \in A_{T}\right)
$$

and assume that one has an auxiliary random variable (r.v.) $Y$ correlated with $Z$ such that $\mathbb{E}[Z \mid Y]$ is available in explicit form. Let $Y^{(1)}, \ldots, Y^{(N)}$ be the sample of $Y$; then, the corresponding unbiased estimator of $\mathbb{E}[Z \mid Y]$ is defined as

$$
\widehat{\pi}_{N}(T):=\frac{1}{N} \sum_{n=1}^{N} \mathbb{E}\left[Z \mid Y^{(n)}\right] .
$$

Note that the variance of this estimator is always less than the variance of the standard $\mathrm{MC}$ one since

$$
\operatorname{Var} Z=\mathbb{E}[\operatorname{Var}[Z \mid Y]]+\operatorname{Var}[\mathbb{E}[Z \mid Y]]
$$

Another popular method for variance reduction is IS. The basic idea of IS is the change of the probability measure, so that the target rare event becomes more likely to occur [10]:

$$
\begin{aligned}
& \pi(T)=\mathbb{E} I\left(X \in A_{T}\right)=\int I\left(x \in A_{T}\right) d \mathbb{P}(x) \\
= & \int I\left(x \in A_{T}\right) \frac{d \mathbb{P}(x)}{d \tilde{\mathbb{P}}(x)} d \tilde{\mathbb{P}}(x)=\tilde{\mathbb{E}}\left[I\left(X \in A_{T}\right) L(X)\right]
\end{aligned}
$$

where $L:=d \mathbb{P}(x) / d \tilde{\mathbb{P}}(x)$ is the likelihood ratio and $\tilde{\mathbb{E}}$ means expectation associated with the probability measure $\tilde{\mathbb{P}}$. Hence, the IS estimator is defined as

$$
\widehat{\pi}_{N}^{\mathrm{IS}}(T):=\frac{1}{N} \sum_{n=1}^{N} I\left(X^{(n)} \in A_{T}\right) L\left(X^{(n)}\right)
$$

where $\left(X^{(1)}, \ldots, X^{(N)}\right)$ are independent and identically-distributed replications generated according to $\tilde{\mathbb{P}}$.

It is well known that the optimal change of measure (zero-variance) requires the knowledge of the probability of interest and, therefore, cannot be practically adopted.

A class of IS estimators (known in the literature as single-twist estimators) can be constructed by shifting the process $X$ with a deterministic path $\eta_{t}(\tilde{\mathbb{P}}$ is the law of $\left.\left\{X_{t}+\eta_{t}\right\}\right)$ in order to make the rare event more likely to occur. In the finite-dimensional case, when $X$ is a centered Gaussian random vector with nondegenerate covariance matrix $\boldsymbol{\Gamma}$, it is easy to show (see, for example, [25]) that the likelihood ratio is given by

$$
L(x)=\exp \left\{-\eta^{\prime} \boldsymbol{\Gamma}^{-1} x+\frac{1}{2} \eta^{\prime} \boldsymbol{\Gamma}^{-1} \eta\right\} .
$$

\section{Bridge Monte-Carlo Estimator}

The BMC is a special case of the conditional MC method, particularly suitable for the estimation of the rare event probabilities in a queueing system with Gaussian input.

Originally proposed by some of the authors in $[14,26,27], \mathrm{BMC}$ is based on the idea of expressing the overflow probability as the expectation of a function of the Bridge $Y:=\left\{Y_{t}\right\}$ of the Gaussian input process $X$, i. e., the process obtained by conditioning $X$ to reach a certain level at some prefixed (deterministic) time instant $\tau$ :

$$
Y_{t}=X_{t}-\psi_{t} X_{\tau}
$$

where $\psi_{t}$ is expressed via the covariance function as

$$
\psi_{t}:=\frac{\Gamma_{t, \tau}}{\Gamma_{\tau, \tau}}
$$

Since the variance of the input is an increasing function of $t$ in all models considered, it is easy to see that $\psi_{t}>0$ for all $t \in T$. Moreover, note that for any $t \in \mathbb{T}, Y_{t}$ is independent of $X_{\tau}$ since

$$
\mathbb{E}\left[X_{\tau} Y_{t}\right]=\Gamma_{\tau, t}-\frac{\Gamma_{t, \tau}}{\Gamma_{\tau, \tau}} \Gamma_{\tau, \tau}=0
$$

and $\left(X_{\tau}, Y_{t}\right)$ has bivariate normal distribution.

The target probability can be expressed in the following form:

$$
\begin{aligned}
\pi(T)=\mathbb{P} & \left(\forall t \in \mathbb{T}: X_{t}>t\right) \\
& =\mathbb{P}\left(\forall t \in \mathbb{T}: X_{\tau}>\frac{t-Y_{t}}{\psi_{t}}\right) \\
& =\mathbb{P}\left(X_{\tau} \geq \sup _{t \in \mathbb{T}} \frac{t-Y_{t}}{\psi_{t}}\right)=\mathbb{P}\left(X_{\tau} \geq \bar{Y}\right)
\end{aligned}
$$

where

$$
\bar{Y}:=\sup _{t \in \mathbb{T}} \frac{t-Y_{t}}{\psi_{t}} .
$$

Observe that random variable $\bar{Y}$ is independent of $X_{\tau}$. For the sake of simplicity, let us prove this property in the case $\mathbb{T}=\{1, \ldots, T\}$ which is enough for simulation needs. Indeed, the random vector $\left(X_{\tau}, Y_{1}, \ldots, Y_{T}\right)$ 
has multivariate normal distribution and, moreover, as it was shown above, $X_{\tau}$ is independent of $Y_{i}, i=1, \ldots, T$; hence, $X_{\tau}$ is independent of the vector $\left(Y_{1}, \ldots, Y_{T}\right)$ (due to the properties of the multivariate normal distribution, see [28] for more details) and, as a consequence, of any function of its components.

Having in mind that $X_{t}={ }_{d} \sqrt{\Gamma_{t, t}} N(0,1)$, the considered probability can be rewritten as follows:

$$
\begin{array}{r}
\pi(T)=\mathbb{P}\left(X_{\tau} \geq \bar{Y}\right)=\int_{R} \mathbb{P}\left(X_{\tau} \geq u\right) \mathbb{P}(\bar{Y} \in d u) \\
=\mathbb{E}\left[\Phi\left(\frac{\bar{Y}}{\sqrt{\Gamma_{\tau, \tau}}}\right)\right]
\end{array}
$$

where independence between $\bar{Y}$ and $X_{\tau}$ is used and $\Phi$ denotes the tail distribution of standard normal variable, that is,

$$
\Phi(x)=\frac{1}{\sqrt{2 \pi}} \int_{x}^{\infty} e^{-y^{2} / 2} d y .
$$

Hence, given a sample $\left\{\bar{Y}^{(n)}, n=1, \ldots, N\right\}$ of $\bar{Y}$, the estimator of $\pi(T)$ is defined as follows:

$$
\widehat{\pi}_{N}^{\mathrm{BMC}}:=\frac{1}{N} \sum_{n=1}^{N} \Phi\left(\frac{\bar{Y}^{(n)}}{\sqrt{\Gamma_{\tau, \tau}}}\right) .
$$

Note that

$$
\Phi\left(\frac{\bar{Y}}{\sqrt{\Gamma_{\tau, \tau}}}\right)=\mathbb{E}\left[I\left(X_{\tau}>\bar{Y}\right) \mid \bar{Y}\right] ;
$$

therefore, the BMC approach is actually a special case of the conditional MC method. By (4), Var $Z$ $\geq \operatorname{Var}[\mathbb{E}[Z \mid \bar{Y}]]$; so, one can expect that the BMC estimator implies variance reduction (with regard to crude MC simulation) in the estimation of the target probability $\pi(T)$.

\section{Simulation Results}

In this section, through simulation results, the accuracy of the BMC estimator and the dependence of its performance on different parameters will be pointed out. For sake of brevity, only the results for FBM input considering $N=10000$ replications (unless otherwise stated) will be presented.

Figure 2 shows the dependence of the target probability on the interval duration $T$ in case of $H=0.8$, a typical value of the Hurst parameter for traffic data. The probability $\pi(T)$ exhibits an exponential decay in agreement with the known LDT asymptotic results (see formula (2)). To better understand the practical applicability of such limits, in Fig. 3, the ratio between BMC estimates and (2) is reported for different values of $T$.
In order to verify the goodness of the present estimator, the dependence of the RE on the parameters $T$ has been considered. Figure 4 highlights that the RE grows slowly and for probabilities of the order of $10^{-11}$, it is still less than $18 \%$, as can be easily verified by comparing the values in Figs. 2 and 4.

The goodness of the present method is also confirmed by the table, where BMC is compared to single twist (with a constant linear drift chosen by minimizing the variance of the estimator) IS in terms of RE: for all

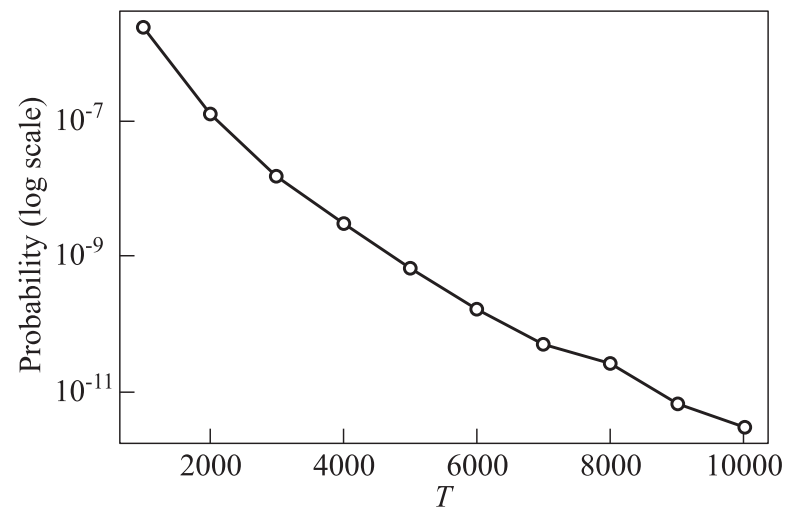

Figure 2 Dependence of $\pi$ on parameter $T$

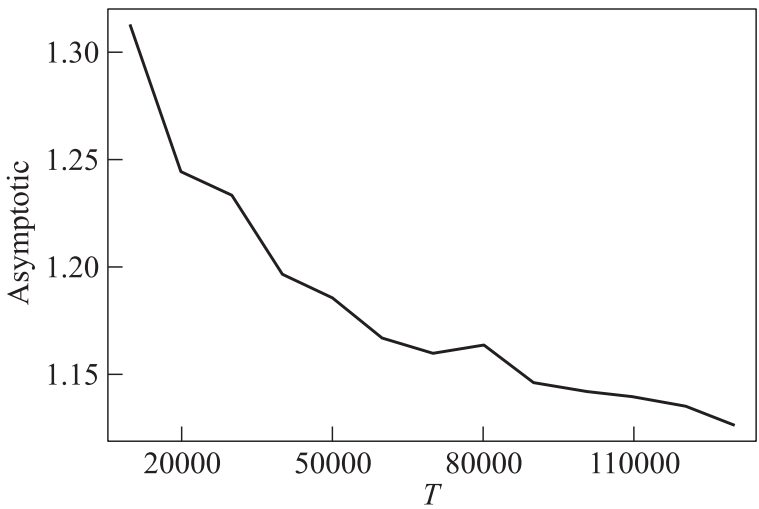

Figure 3 Comparison with LDT asymptotic results

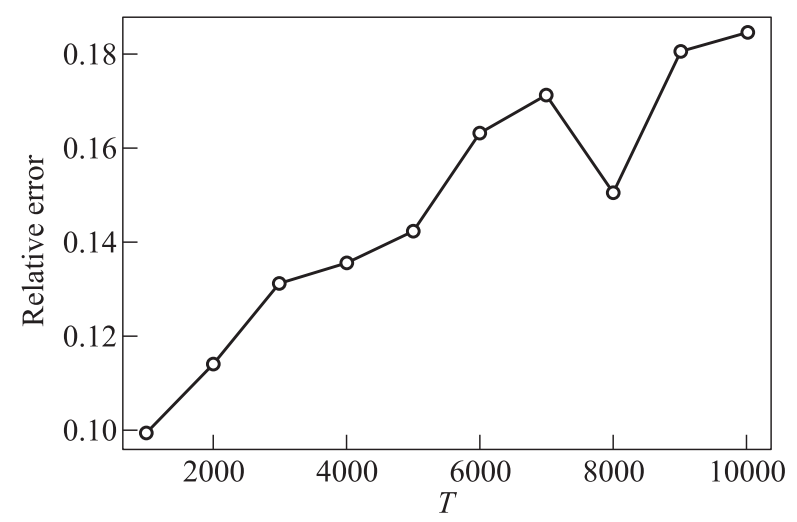

Figure 4 Dependence of the RE on $T$ 
Relative errors for BMC and IS

\begin{tabular}{|c|c|c|}
\hline$T$ & BMC & IS \\
\hline 200 & 0.0691 & 0.1483 \\
400 & 0.0779 & 0.1850 \\
600 & 0.0941 & 0.1755 \\
800 & 0.0894 & 0.1772 \\
1000 & 0.1039 & 0.2275 \\
1200 & 0.1044 & 0.2084 \\
1400 & 0.1101 & 0.1980 \\
1600 & 0.1104 & 0.2462 \\
1800 & 0.1150 & 0.2182 \\
2000 & 0.1177 & 0.2696 \\
\hline
\end{tabular}

the considered values of $T, \mathrm{BMC}$ reduces the RE by a factor around 2 .

To better understand the asymptotic properties of the estimator (at least heuristically), in Figs. 5 and 6, the behavior of $N_{T}$ and $R_{T}$ is shown: the required number of sample paths (for a fixed value of the RE) grows very slowly (at least in logarithmic scale) and the relative efficiency is above 1.9 for $T>20000$ (and gets closer to 2 for higher values of $T$ ).

In all previous simulation sets, the conditioning point $\tau$ in the BMC algorithm has been assumed equal

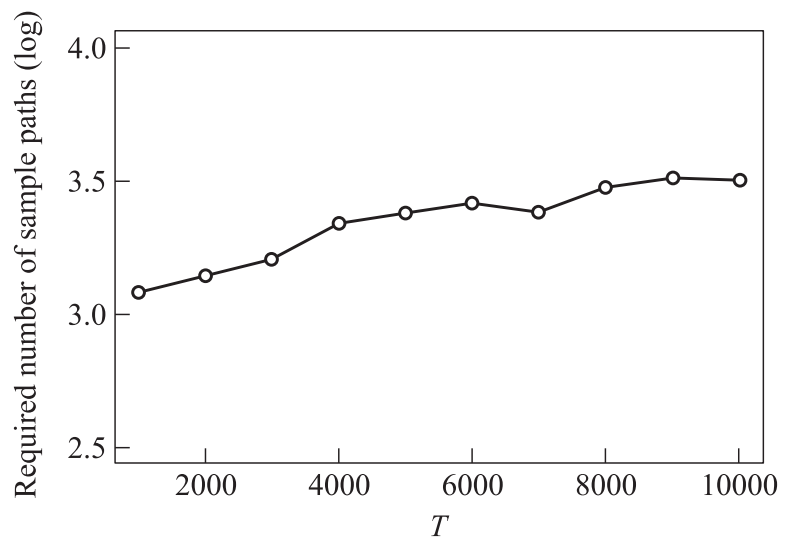

Figure 5 Dependence of $N_{T}$ on $T$ for $\mathrm{RE}_{\max }=0.1$

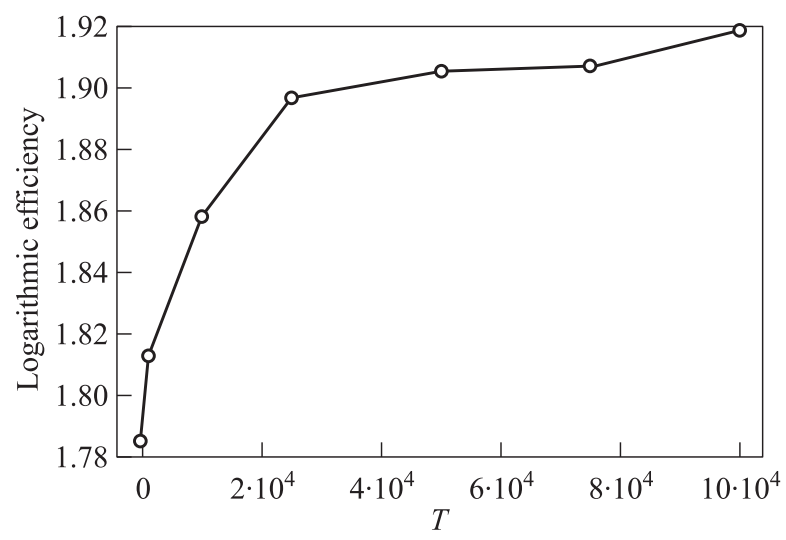

Figure 6 Dependence of $R_{T}$ on $T$ for $N=10000$

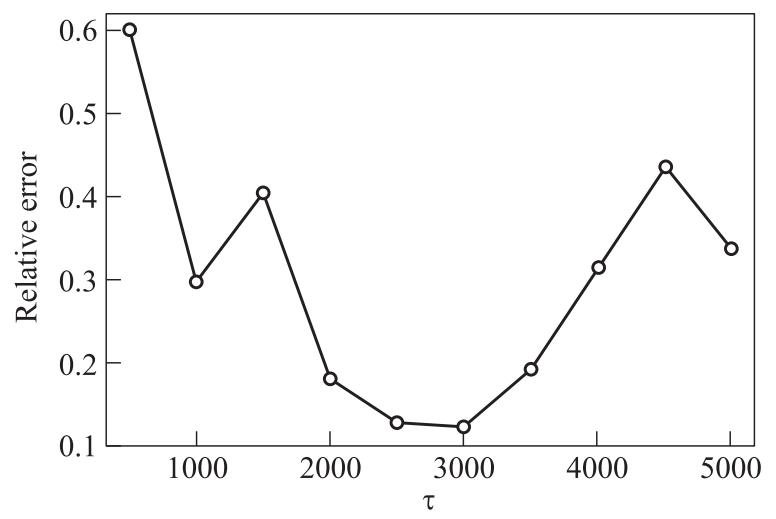

Figure 7 Dependence of RE on $\tau$ for $T=3000$

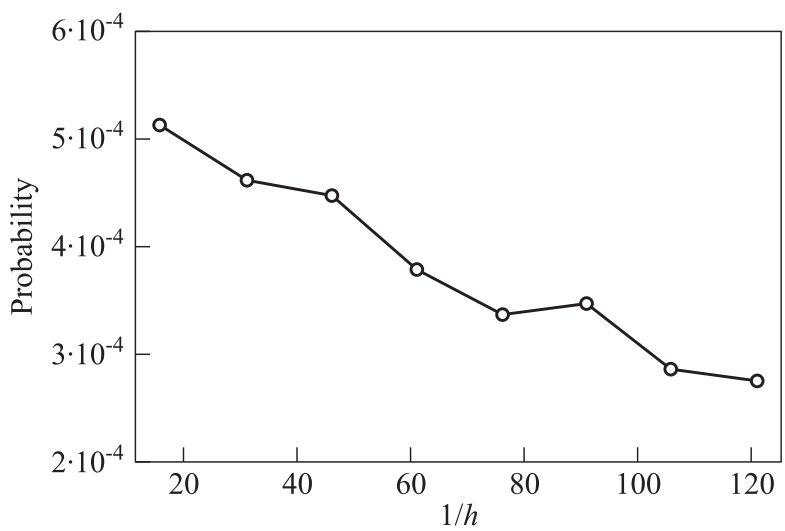

Figure 8 Effect of the discretization step $h$

to the duration of the interval. The correctness of such choice is confirmed for $T=3000$ by Fig. 7 in which an absolute minimum of the RE can be identified in a neighborhood of $T$.

Finally, in Fig. 8, the effect of the discretization step (simulations always involve finite-size vectors and not continuous-time processes!) on the estimated probability is highlighted. In more detail, $T=100$ with discretisation step $h$ (in the previous simulations, $h=1$ ) has been considered. This means that each FBM sample path consists of $T / h$ points: apart some oscillations of the estimated value (the confidence intervals should also be taken into account!), as expected, the target probability decreases when sampling is more dense.

\section{Concluding Remarks}

In this paper, the estimation of the busy period duration in Gaussian queues was considered with focus on the upper tail of the distribution. To address the issues related to the simulation of such rare events, the authors considered a special case of conditional MC estimator based on bridge processes. In more detail, the BMC 
approach exploits the Gaussian nature of the input process (independence is equivalent to uncorrelatedness) and relies on the properties of bridges to write down the target probability as the conditional one.

To study the properties of the proposed estimator, several simulation experiments have been carried out focusing on FBM sample paths, although the methods are applicable to any Gaussian process with increasing variance. In the experimental analysis, different values of the relevant parameters (duration of the interval, choice of the conditioning point, and discretization step) have been considered and the asymptotic properties of the estimator (in terms of relative efficiency and duration of the simulation for a given precision of the estimates) have been investigated. Finally, it is worth mentioning that the relative error is halved with respect to single twist IS, highlighting the efficiency of BMC over well-known rare event simulation techniques.

\section{Acknowledgments}

This work is supported by the Russian Foundation for Basic Research, projects 15-07-02341, 15-07-02354, and 15-07-02360 and also by the Program of Strategic Development of Petrozavodsk State University.

\section{References}

1. Leland, W. E., M. S. Taqqu, W. Willinger, and D. V. Wilson. 1994. On the self-similar nature of Ethernet traffic (extended version). IEEE ACM Trans. Network. 2(1):1-15.

2. Norros, I. 1995. On the use of fractional Brownian motion in the theory of connectionless networks. IEEE J. Sel. Area. Comm. 13(6):953-962.

3. Mandjes, M. 2007. Large deviations of Gaussian queues. Chichester: Wiley. 340 p.

4. Erramilli, A., O. Narayan, and W. Willinger. 1996. Experimental queueing analysis with long-range dependent packet traffic. IEEE ACM Trans. Network. 4(2):209-223.

5. Samorodnitsky, G. 2007. Long range dependence. Found. Trends ${ }^{R}$ Stochastic Syst. 1(3):163-257. doi: 10.1561/0900000004.

6. Allman, M., V. Paxson, and E. Blanton. 2009. TCP Congestion Control. RFC 5681 (Draft Standard).

7. Kouvatsos, D. D. 2000. Performance evaluation and applications of ATM networks. Kluwer Academic. 472 p.

8. Ross, S. M. 2006. Simulation. Elsevier. 314 p.

9. Ganesh, A., N. O'Connell, and D. Wischik. 2004. Big queues. Lecture notes in mathematics ser. Springer. 260 p.

10. Heidelberger, P. 1995. Fast simulation of rare events in queueing and reliability models. ACM Trans. Model. Comput. Simul. 5(1):43-85.

11. Glasserman, P., and Y. Wang. 1997. Counterexamples in importance sampling for large deviations probabilities. Ann. Appl. Probab. 7(3):731-746.
12. Lukashenko, O. V., E. V. Morozov, and M. Pagano. 2016. On Conditional Monte Carlo estimation of busy period probabilities in Gaussian queues. Comm. Com. Inf. Sc. 601:280-288. doi: 10.1007/978-3-319-30843-2_29.

13. Lukashenko, O. V., E. V. Morozov, and M. Pagano. 2016. On the use of a bridge process in a Conditional Monte Carlo simulation of Gaussian queues. Comm. Com. Inf. Sc. 638:207-220. doi: 10.1007/978-3-319-44615-8_18.

14. Giordano, S., M. Gubinelli, and M. Pagano. 2005. Bridge Monte-Carlo: A novel approach to rare events of Gaussian processes. 5th St. Petersburg Workshop on Simulation Proceedings. 281-286.

15. Norros, I. 1999. Busy periods of fractional Brownian storage: A large deviations approach. Adv. Perf. Anal. 2:1-19.

16. Mandjes, M., I. Norros, and P. Glynn. 2009. On convergence to stationarity of fractional Brownian storage. Ann. Appl. Probab. 19:1385-1403.

17. Taqqu, M. S., W. Willinger, and R. Sherman. 1997. Proof of a fundamental result in self-similar traffic modeling. Comput. Commun. Rev. 27:5-23.

18. Addie, R., P. Mannersalo, and I. Norros. 2002. Most probable paths and performance formulae for buffers with Gaussian input traffic. Eur. Trans. Telecommun. 13:183196.

19. Kulkarni, V., and T. Rolski. 1994. Fluid model driven by an Ornstein-Uhlenbeck process. Probab. Eng. Inform. Sc. 8:403-417.

20. Deuschel, J. D., and D. W. Stroock. 1989. Large deviations. Academic Press. 330 p.

21. Mandjes, M., P. Mannersalo, I. Norros, and M. van Uitert. 2006. Large deviations of infinite intersections of events in Gaussian processes. Stoch. Proc. Appl. 116:1269-1293.

22. Dieker, A. B. 2005. Conditional limit theorems for queues with Gaussian input: A weak convergence approach. Stoch. Proc. Appl. 115(5):849-873.

23. Dieker, A. B., and M. Mandjes. 2005. On asymptotically efficient simulation of large deviation probabilities. $A d v$. Appl. Probab. 37:539-552.

24. Dieker, A. B., and M. Mandjes. 2006. Fast simulation of overflow probabilities in a queue with Gaussian input. ACM Trans. Model. Comput. Simul. 16:119-151.

25. Asmussen, S., ans P. Glynn. 2007. Stochastic simulation: Algorithms and analysis. Springer. $476 \mathrm{p}$.

26. Giordano, S., M. Gubinelli, and M. Pagano. 2007. Rare events of Gaussian processes: A performance comparison between Bridge Monte-Carlo and Importance Sampling. Next generation teletraffic and wired/wireless advanced networking. Eds. Y. Koucheryavy, J. Harju, and A. Sayenko. Computer communication networks and telecommunications ser. Berlin-Heidelberg: Springer. 4712:269-280.

27. Lukashenko, O. V., E. V. Morozov, and M. Pagano. 2012. Performance analysis of Bridge Monte-Carlo estimator. Transactions of KarRC RAS 5:54-60.

28. Gut, A. 2009. An intermediate course in probability. Springer. 304 p. 


\title{
Contributors
}

L ukashenko Oleg V. (b. 1986) - Candidate of Science (PhD) in physics and mathematics, scientist, Institute of Applied Mathematical Research of Karelian Research Centre of the Russian Academy of Sciences, 11 Pushkinskaya Str., Petrozavodsk 185910, Republic of Karelia, Russian Federation; lecturer, Petrozavodsk State University, 33 Lenin Str., Petrozavodsk 185910, Republic of Karelia, Russian Federation; lukashenko@krc.karelia.ru

Morozov Evsei V. (b. 1947) - Doctor of Science in physics and mathematics, professor, leading scientist, Institute of Applied Mathematical Research of Karelian Research Centre of the Russian Academy of Sciences, 11 Pushkinskaya Str., Petrozavodsk 185910, Republic of Karelia, Russian Federation; professor, Petrozavodsk State University, 33 Lenin Str., Petrozavodsk 185910, Republic of Karelia, Russian Federation; emorozov@karelia.ru

Pagano Michele (b. 1968) - PhD in Information Engineering, associate professor, University of Pisa, 43 Lungarno Pacinotti, Pisa 56126, Italy; m.pagano@iet.unipi.it

\section{ОБ ЭФФЕКТИВНОСТИ ОЦЕНКИ МОНТЕ КАРЛО НА ОСНОВЕ ГАУССОВСКОГО МОСТА*}

\author{
О. В. Лукашенко ${ }^{1,2}$, Е. В. Морозов ${ }^{1,2}$, М. Пагано ${ }^{3}$ \\ ${ }^{1}$ Институт прикладных математических исследований Карельского научного центра Российской акаде- \\ мии наук \\ ${ }^{2}$ Петрозаводский государственный университет \\ ${ }^{3}$ Университет г. Пиза, Италия
}

\begin{abstract}
Аннотация: Наличие долговременной зависимости в современных сетях передачи данных приводит к тому, что объем передаваемого трафика может быть большим на протяжении значительного периода времени. Это, в свою очередь, влечет перегрузку систем на протяжении длительного периода времени. В данной работе рассматривается задача оценки вероятности занятости системы обслуживания с гауссовским входным потоком в течение некоторого заданного периода $T$. При больших значениях $T$ интересующее нас событие является редким, и для оценки его вероятности с приемлемой точностью необходимо использовать специальные методы понижения дисперсии оценки. В статье рассмотрен частный случай условного метода Монте Карло, который заключается в том, что искомая вероятность может быть выражена как математическое ожидание некоторой функции от так называемого гауссовского моста. Исследована эффективность предложенной процедуры, а также влияние шага дискретизации на свойство получаемой оценки.
\end{abstract}

Ключевые слова: гауссовские процессы; условный метод Монте Карло; процесс моста; редкие события; уменьшение дисперсии

DOI: $10.14357 / 19922264170202$

\section{Литература}

1. Leland W. E., Taqqu M.S., Willinger W., Wilson D. V. On the self-similar nature of Ethernet traffic (extended version) // IEEE ACM Trans. Network., 1994. Vol. 2. No. 1. P. $1-15$.

2. Norros I. On the use of fractional Brownian motion in the theory of connectionless networks // IEEE J. Sel. Area. Comm., 1995. Vol. 13. No. 6. P. 953-962.

3. Mandjes M. Large deviations of Gaussian queues. Chichester: Wiley, 2007. 340 p.

4. Erramilli A., Narayan O., Willinger W. Experimental queueing analysis with long-range dependent packet traf- fic // IEEE ACM Trans. Network., 1996. Vol. 4. No. 2. P. 209-223.

5. Samorodnitsky G. Long range dependence // Found. Trends ${ }^{\circledR}$ Stochastic Syst., 2007. Vol. 1. No. 3. P. 163257. doi: $10.1561 / 0900000004$.

6. Allman M., Paxson V., Blanton E. TCP congestion control. RFC 5681 (Draft Standard), 2009.

7. Kouvatsos D. D. Performance evaluation and applications of ATM networks. - Kluwer Academic, 2000. 472 p.

8. Ross S. M. Simulation. - Elsevier, 2006. 314 p.

9. Ganesh A., O'Connell N., Wischik D. Big queues. - Lecture notes in mathematics ser. - Springer, 2004. 260 p.

* Работа поддержана грантами РФФИ №№ 15-07-02341, 15-07-02354 и 15-07-02360, а также программой стратегического развития Петрозаводского государственного университа. 
10. Heidelberger $P$. Fast simulation of rare events in queueing and reliability models // ACM Trans. Model. Comput. Simul., 1995. Vol. 5. No. 1. P. 43-85.

11. Glasserman P., Wang $Y$. Counterexamples in importance sampling for large deviations probabilities // Ann. Appl. Probab., 1997. Vol. 7. No. 3. P. 731-746.

12. Lukashenko O. V., Morozov E. V., Pagano M. On Conditional Monte Carlo estimation of busy period probabilities in Gaussian queues // Comm. Com. Inf. Sc., 2016. Vol. 601. P. 280-288. doi: 10.1007/978-3-319-308432_29.

13. Lukashenko O.V., Morozov E.V., Pagano M. On the use of a bridge process in a Conditional Monte Carlo simulation of Gaussian queues // Comm. Com. Inf. Sc., 2016. Vol. 638. P. 207-220. doi: 10.1007/978-3-31944615-8_18.

14. Giordano S., Gubinelli M., Pagano M. Bridge Monte-Carlo: A novel approach to rare events of Gaussian processes // 5th St. Petersburg Workshop on Simulation Proceedings, 2005. P. 281-286.

15. Norros I. Busy periods of fractional Brownian storage: A large deviations approach // Adv. Perf. Anal., 1999. Vol. 2. P. 1-19.

16. Mandjes M., Norros I., Glynn P. On convergence to stationarity of fractional Brownian storage // Ann. Appl Probab., 2009. Vol. 19. P. 1385-1403.

17. Taqqu M.S., Willinger W., Sherman R. Proof of a fundamental result in self-similar traffic modeling // Comput Commun. Rev., 1997. Vol. 27. P. 5-23.

18. Addie R., Mannersalo P., Norros I. Most probable paths and performance formulae for buffers with Gaussian input traffic // Eur. Trans. Telecommun., 2002. Vol. 13. P. 183-196.
19. Kulkarni V., Rolski T. Fluid model driven by an OrnsteinUhlenbeck process // Probab. Eng. Inform. Sc., 1994. Vol. 8. P. 403-417.

20. Deuschel J. D, Stroock D. W. Large deviations. - Academic Press, 1989. 330 p.

21. Mandjes M., Mannersalo P., Norros I., van Uitert M. Large deviations of infinite intersections of events in Gaussian processes // Stoch. Proc. Appl., 2006. Vol. 116. P. 12691293.

22. Dieker A. B. Conditional limit theorems for queues with Gaussian input: A weak convergence approach // Stoch. Proc. Appl., 2005. Vol. 115. No. 5. P. 849-873.

23. Dieker A. B., Mandjes M. On asymptotically efficient simulation of large deviation probabilities // Adv. Appl. Probab., 2005. Vol. 37. P. 539-552.

24. Dieker A. B., Mandjes M. Fast simulation of overflow probabilities in a queue with Gaussian input // ACM Trans. Model. Comput. Simul., 2006. Vol. 16. P. 119-151.

25. Asmussen S., Glynn P. Stochastic simulation: Algorithms and analysis. - Springer, 2007. $476 \mathrm{p}$.

26. Giordano S., Gubinelli M., Pagano M. Rare events of Gaussian processes: A performance comparison between Bridge Monte-Carlo and importance sampling // Next generation teletraffic and wired/wireless advanced networking / Eds. Y. Koucheryavy, J. Harju, and A. Sayenko. - Computer communication networks and telecommunications ser. - Berlin-Heidelberg: Springer, 2007. Vol. 4712. P. 269-280.

27. Lukashenko O. V., Morozov E. V., Pagano M. Performance analysis of Bridge Monte-Carlo estimator // Труды Карельского НЦ РАН, 2012. Т. 5. С. 54-60.

28. Gut A. An intermediate course in probability. - Springer, 2009. $304 \mathrm{p}$. 